\title{
Regional Therapies for Advanced Cancer
}

\author{
K. K. Turaga and T. C. Gamblin, MD, MS \\ Division of Surgical Oncology, Department of Surgery, Medical College of Wisconsin, Milwaukee, WI
}

Regional therapies for advanced malignancies have evolved from being merely anatomically targeted therapies to individualized therapies that are evidence based and discerning of the patients who would derive the most benefit. Pursuits of precise surgical technique, as well as advances in anesthesia and systemic therapies, have allowed the application of these therapies in new frontiers.

In this issue of Annals of Surgical Oncology, selected peer-reviewed papers presented at the eighth international regional therapies symposium have been chosen to demonstrate the strides made in the last few years while highlighting the challenge of achieving durable responses for patients with advanced cancer.

The first article, by Kwa and Muggia, is a historical review of trials of intraperitoneal chemotherapy for patients with ovarian malignancies. ${ }^{1}$ Despite landmark trials such as the GOG 172, which clearly showed survival benefit for patients receiving regional therapy with intraperitoneal paclitaxel, there appears to a relative reluctance of practitioners to adopt this technique widely. The authors discuss the reasons for this challenge, highlighting opportunities for adoption of hyperthermic intraperitoneal chemotherapy (HIPEC) for effective delivery of regional therapy without the associated myriad issues with intraperitoneal chemotherapy.

The next two articles explore further the understanding of tumor biology, the microenvironment, and implications for regional therapies. Speicher et al. ${ }^{2}$ present a novel study of using optical spectroscopy and EF35 in evaluating hypoxia in patients with intransit melanoma undergoing isolated limb infusions (ILI). Hypoxia is believed to play an important role in chemotherapy, and radiation and has been implicated as a

(C) Society of Surgical Oncology 2014

First Received: 8 January 2014;

Published Online: 25 February 2014

T. C. Gamblin, MD, MS

e-mail: tcgamblin@mcw.edu potentiating factor for responses to ILI versus limb perfusions, but measurement of the degree of hypoxia is poorly reported. By using optical spectroscopy, tumor hypoxia was measured as being more profound than in the normal skin of patients undergoing ILI. This technique could help noninvasively examine the tissue microenvironment and provide clues to the effectiveness of therapies.

Lohani et al. ${ }^{3}$ present their research examining the microenvironment regarding patients with the pseudomyxoma peritonei syndrome; their work examines cytokines in the serum and ascites. They also attempt to correlate these levels with the stroma and tumor cells. While C-reactive protein is elevated in patients with pseudomyxoma peritonei, traditional inflammatory markers such as IL-1 were not elevated. This work supports a complex model of inflammation and neoplasia demonstrating the peritoneal synthesis of cytokines and serum elevations of IL- 8 and MIP- $1 \beta$.

While understanding that tumor-host microenvironments are important in advancing the field, the direct impact of these interactions is seen in patients who undergo regional therapies. The next articles demonstrate the delicate balance between aggressiveness of disease, genetics, and histology in predicting response to therapy.

Magge et al. ${ }^{4}$ tackle a difficult problem in evaluating the role of cytoreductive surgery and HIPEC in the management of patients with gastric cancer and peritoneal carcinomatosis. Close on the heels of a recently published randomized trial, this study demonstrates the survival benefit in selected patients with gastric cancer in a Western population. ${ }^{5}$ Despite aggressive surgical techniques and careful selection of patients with minimal peritoneal disease [median peritoneal carcinomatosis index $(\mathrm{PCI})=10$ ], the 3 -year survival in this cohort was a humbling $18 \%$. The group identified characteristics such as male gender, signet ring histology, incomplete cytoreduction, and multivisceral surgery as factors predicting either worse survival or progression of disease. 
Acknowledging that signet ring histology carries a worse prognosis, Winer et al. ${ }^{6}$ sought to identify patients who would potentially benefit from aggressive cytoreduction. This article highlights two critical concepts: first, appendiceal and colorectal tumors are inherently different, with varied biologies; and second, complete cytoreduction is critical in providing patients with the longest survival, especially in patients with colorectal primary disease. They also found that patients with lean body mass indices (BMI) $\left(<25 \mathrm{~kg} / \mathrm{m}^{2}\right)$ also did worse, suggesting the adverse effect of cancer- and therapy-related cachexia.

McPartland and Goodman ${ }^{7}$ looked at patients stratified by BMI groups and found that patients that were either underweight or obese $\left(>30 \mathrm{~kg} / \mathrm{m}^{2}\right)$ did not have different outcomes than patients with normal BMI. Obese patients had their tumors diagnosed in a timely fashion and had a similar burden of disease as patients in other subgroups. This article is provocative because a third of the US population is obese. The differences between the two studies for patients with low BMI could be from the heterogeneity of histology.

Although host issues can certainly play an important role in the perioperative outcome of patients, Kwa et al. ${ }^{8}$ present a thoughtful study examining the role of genetic alterations in prediction of outcomes. In their series of 62 patients treated for advanced epithelial ovarian cancer with intraperitoneal cisplatin, the authors noted that patients with deleterious $B R C A$ mutations did well with improved survival. The paradigm of using genetic mutations to predict outcomes in these patients deserves further investigation.

Advances in medical care have occurred as a result of pioneers who have continued to forge new paths in managing patients with advanced disease. Wong et al. ${ }^{9}$ present a unique study that examined one of the largest institutional series of patients undergoing ILIs for melanoma and compared patients who underwent excision of residual melanoma after an ILI with patients who experienced complete response. The group reported equivalent median overall survival, suggesting that regional therapy, even as a neoadjuvant/conversion strategy, might be beneficial for patients with locally aggressive disease.

One of the important problems faced in advanced peritoneal disease is the occurrence of malignant ascites, and Randle et al. ${ }^{10}$ present their experience in controlling ascites in patients undergoing cytoreductive surgery and HIPEC. Despite having a mixed subset of histologies, the authors reported a 3-month $93 \%$ ascites-free survival, which was feasible even in patients who underwent an R2 resection $(84 \%)$.

Wong et al. ${ }^{11}$ explore an alternative methodology of managing patients with recurrent mesothelioma with repeat cytoreductive surgery and HIPEC in addition to early postoperative intraperitoneal chemotherapy. In their series of 29 patients, 8 underwent repeated HIPEC for recurrent disease, with a significant survival benefit of almost 80 months compared to those who did not receive a second HIPEC. This study returns to the question of tumor-host interaction; although it is hard to determine whether this is the effect of tumor biology or aggressive therapy, careful selection of patients with mesothelioma can yield good results.

Aggressive surgical therapy needs excellent anesthetic support, and the study from Owusu-Agyemang et al. ${ }^{12}$ examines the use of epidural analgesia in 215 adult patients undergoing HIPEC. This study used the practice of starting the epidural before the HIPEC and found a significant reduction in the blood loss and the fluid requirements of these patients. Given the complexities of managing fluids in this group of patients, this study provides evidence of potential improvement in perioperative care.

Reduction in blood transfusions might be more important than one thinks, and the next study, by Bartlett et al., ${ }^{13}$ examines a national database (National Surgical Quality Improvement Program, NSQIP) capturing data on patients undergoing cytoreductive surgery and HIPEC. Measuring perioperative outcomes and improvement in the surgical quality of patients is critical in ensuring better overall outcomes, and this study identifies patients who might be at increased risk. The relatively low mortality rate $(2.3 \%)$ and morbidity rate $(31 \%)$ are acceptable. Malnutrition, increased operative time, and performance of gastrectomy in addition to blood transfusion were associated with increased mortality and morbidity.

Although the articles in this series demonstrate the significant advances made in tumor-host interactions, surgical and anesthetic care, and patient prognostication, collaboration is the key to significant advances in this field. The American Society of Peritoneal Surface Malignancies presents their consensus guidelines for standardization of HIPEC delivery in the United States to increase collaboration among centers and uniformity of the care. ${ }^{14}$ This provides a platform for delivery of care to patients while also stimulating collaborative research potentials.

There has never been a more exciting period of time for the application of regional therapies for patients with advanced malignancies, and the eighth international symposium for regional therapies offers a snapshot of advances in this field. These articles highlight our improving understanding of tumor-host interactions, therapeutics, and prognostics; we hope the results will spur more research and collaboration, which are essential to progress.

CONFLICT OF INTEREST The authors declare no conflict of interest. 


\section{REFERENCES}

1. Kwa M, Muggia F. Ovarian cancer: a brief historical overview of intraperitoneal trials. Ann Surg Oncol. In press.

2. Speicher PJ, et al. Hypoxia in melanoma: using optical spectroscopy and EF5 to assess tumor oxygenation before and during regional chemotherapy for melanoma. Ann Surg Oncol. In press.

3. Lohani K, et al. Pseudomyxoma peritonei: inflammatory responses in the peritoneal microenvironment. Ann Surg Oncol. In press.

4. Magge D, et al. Aggressive locoregional surgical therapy for gastric peritoneal carcinomatosis. Ann Surg Oncol. In press.

5. Yang XJ, et al. Cytoreductive surgery and hyperthermic intraperitoneal chemotherapy improves survival of patients with peritoneal carcinomatosis from gastric cancer: final results of a phase III randomized clinical trial. Ann Surg Oncol. 2011;18: 1575-81.

6. Winer $\mathrm{J}$, et al. Impact of aggressive histology and location of primary tumor on the efficacy of surgical therapy for peritoneal carcinomatosis of colorectal origin. Ann Surg Oncol. In press.

7. McPartland SJ, Goodman MD. The effect of elevated body mass index on outcomes following cytoreductive surgery with hyperthermic intraperitoneal chemotherapy. Ann Surg Oncol. In press.

8. Kwa M, et al. Ovarian cancer in BRCA mutation carriers: improved outcome after intraperitoneal (IP) cisplatin. Ann Surg Oncol. In press.
9. Wong $\mathbf{J}$, et al. Resection of residual disease after isolated limb infusion (ILI) is equivalent to a complete response after ILI-alone in advanced extremity melanoma. Ann Surg Oncol. In press.

10. Randle RW, et al. Efficacy of cytoreductive surgery with hyperthermic intraperitoneal chemotherapy in the management of malignant ascites. Ann Surg Oncol. In press.

11. Wong J, et al. Repeat cytoreductive surgery and heated intraperitoneal chemotherapy may offer survival benefit for intraperitoneal mesothelioma: a single institution experience. Ann Surg Oncol. In press.

12. Owusu-Agyemang $P$, et al. Safety of epidural analgesia in the perioperative care of patients undergoing cytoreductive surgery with hyperthermic intraperitoneal chemotherapy. Ann Surg Oncol. In press.

13. Bartlett EK, et al. Morbidity and mortality of cytoreduction with intraperitoneal chemotherapy: outcomes from the ACS NSQIP Database. Ann Surg Oncol. In press.

14. Turaga K, et al. Consensus guidelines from the American Society of Peritoneal Surface Malignancies on Standardizing the Delivery of Hyperthermic Intraperitoneal Chemotherapy (HIPEC) in colorectal cancer patients in the United States. Ann Surg Oncol. In press. 